\title{
Will Information-Communications Systems Survive? Web 2.x and SocNet as Placebos or Real Internet Survival Kit
}

\author{
Prof.dr.sc. Fjodor Ruzic \\ Institute for Informatics, Zagreb, Croatia
}

Fjodor.Ruzic@open.hr

\begin{abstract}
Since the information-communications systems lay on three interactive portions of infrastructure (network, information bases, devices/interfaces), the current Internet domination of Web 2.x will be finished very soon. The Internet as we are using today has to be changed. In particular, the next generation of Internet has to be more user friendly, information oriented and computerless interfaced. It should add features that will help users utilize it in the same way they utilize other methods of communication and transport and have the same or superior level of flexibility. What will be with Internet if it would not answer on new demand for greater and more flexible/mobile communication? Some projections are based on presumptions that Internet will not survive. In contrary, there are also presumptions that Internet could survive with Web 2.x and Social Networks development and with IPv6 as well. Does it means real base and solution for helping Internet to stay alive or it is only placebo for today's Internet users. In order to reshape current Internet status, some Web 2.x and Social Networks deployments are described in this paper as well as the assumptions on the structure of survival kit for Internet to stay alive in the future.
\end{abstract}

Ke ywords: Information-Communications Systems, Internet, Web, Social Networks, Unified Communications

\section{Introduction}

Web and its infrastructure is evolving from its beginning into widespread mechanism of communication and for sharing ideas amongst individuals and various socials and business groups.

Nowadays we are faced with the questions about Internet capability to serve this enormous development process continued with the more mobile and ubiquitous computing facilities. With the Web 2.x technology deployment and the social networks diversification, the Internet has got new power to serve people and the community. However, there is a potential challenge caused by the

Internet capability to stay the universal

Material published as part of this publication, either on-line or in print, is copyrighted by the Informing Science Institute.

Permission to make digital or paper copy of part or all of these works for personal or classroom use is granted without fee provided that the copies are not made or distributed for profit or commercial advantage AND that copies 1) bear this notice in full and 2) give the full citation on the first page. It is permissible to abstract these works so long as cred it is given. To copy in all other cases or to republish or to post on a server or to redistribute to lists requires specific permission and payment of a fee. Contact Publisher@InformingScience.org to request redistribution permission. infrastructure for the future demand in the information-communications systems development process. This paper gives insight into existing and future Web technology deployment accompanied $w$ ith the social networks. Web 2.x and current social networks could be the instruments to make Internet alive. This paper discusses the Web 2.x and Social Networks as the real tool for making 
Internet useful for the future demand. Besides these categories, the terms of cloud computing, Web 3.x technology and new Social Networks concepts are described as the issues acting as promoter for the new form of Internet survival kit. That is why the Web 2.x and current social networks could be thinking as the placebo making the user sense that this is the full potential of the Internet. However, such presumption could lead to the end of the Internet that has to be changed dramatically in order to stay dominant infrastructure and media category. Since the future of any information-communications systems is strongly dependent on the communications system, the Internet to survive has to be modified into truly universal communications environment.

\section{Basic Notions on Information-Communications Systems Development}

Information is economic resource and the power, but information that cannot be readily moved is gridlock on the net. Immobile information makes our businesses larger, more static and hierarchical than they need to be. It makes our economies less flexible, our jobs less fulfilling, and our lives less luminous with opportunity. The computer era is falling before the one technological force that could surpass in impact the computer's ability to process information. That is communication, which is more essential to our humanity. Communication is the way we weave together a personality, a family, a business, a nation and a world. As Guilder (2002) states the Telecosm will make human communication universal, instantaneous, unlimited in capacity and free at the margins.

In most working environment, people currently appear to work by doing four things: they look information, they search for things, they receive information, and they subscribe to things. They communicate with each other using various forms of communication. As a result, they transact business within the enterprise, with partners and supply chain, and with customers. When business are coping with the Internet, mobility and the enterprise appears to be the need for collaboration within and across enterprises. Initially, the Internet was primarily utilized as a medium for communication. After the initial stage, many organizations and individuals began to discover that they could utilize the Internet for information activities. The early application was the World Wide Web (Web) as a hypertext tool allowing computers to link information. In the same time, despite all its promises, the Web-enabled technologies are not free of associated risks that caused new Web technologies development as well as conceptual rethinking of the Internet itself. Web 2.x is undoubtedly a set of economic, social and technology issues that collectively form the basis for the one generation of the Internet - the Internet 2.x. The Internet makes reformation activities creating new fundamentals of and for our world. This reformation creates changes that are deeply cultural and not merely social and economic. Web 2.x as participation-platform spins the issues that are making the basis for Web 3.x development.

These notions are based on the usage of the social network as a meta-layer above both the Web and the mobile domains. The social network becomes the interface between various departments and also across enterprises. Most of improvements in overall enterprise computing would be done with the Web 3.x. Web 3.x is going to deliver a new generation of business applications that will see business computing converge on the same fundamental on-demand architecture as consumer applications, and it promises yet another Internet revolution. It would provide the foundation for systems that can reason in a human fashion. New information-communications systems thus promises to be much more useful than recent Web 2.x sites and to render today's search engines more or less obsolete. The information-communications systems in the future should involve the disintegration of digital data and software into modular components (distribution and decentralization) that, through the use of simple tools, can be reintegrated into new applications or functions on the fly by either machines or people. These processes ask for new Internet communica- 
tion architecture deployed through the Internet 3.x or New Generation Network (NGN) infrastructure. This scenario includes the generalized inter-networking architecture that connects the whole communications and transportation infrastructure (wired and cellular phone networks, wired and wireless data networks, optical and satellite infrastructure, etc.) This process tends to the Unif ied Communications concept of real-time communications. By integrating various forms of communication, such as voice, video, instant messaging, conferencing, presence and voicemail, individuals as well as enterprises can more effectively improve the control and management of the communication processes. When these two 3.x categories (Web 3.x and Internet 3.x) become fully converged, then Internet will have chances to survive. The real success will be reached when triple-W factor model would be set applying: What people want, Where they want, and When they need (WWW InCo Systems).

The future information-communications systems based on NGN foster emerging applications and technologies that creates the arena for:

- The mobile environment with universal connectivity in which any piece of information is available anytime and anywhere

- The converged environment where the computing and networking are a natural part of everybody's life and activities in the manner of invisibility

The architecture for new information-communications systems gives the invisibility of the technologies - opening the era of ubiquitous computing, or the age of universal technology, when technology recedes invisibly into the background of our lives.

\section{Web 2.x and its Deployment}

The Web 2.x is dealing greatly with the concept of the Web as a communication media. Web 2.x is also stated as the Participatory Web based on architecture for interaction. As Friedman (2007) noted, the Web 2.x changed Internet community from "Open-Sourcing" (Self-Organizing Collaborative Communities) to "Uploading" (Online Communities, Open Source, Blogging, Wikipedia and social networks).

O'Reilly (2005) discusses the many aspects of Web 2.0. The discussion includes exploring the technologies of AJAX, Web Services and other means for making Web content more dynamic. In the discussion of Web 2.0, O'Reilly tends to focus on the technologies and not as much on the social phenomena underlying Web 2.0 applications. In the past few years, however, it has become increasingly clear that the social networking construction is critical to the success of Web 2.0 applications. The fact that sharing of content can be enhanced by personal connections, rather than primarily via search or other query techniques has emerged as a major aspect of successful Web 2.0 applications. Web 2.0 is generally regarded as the social Web - the Internet of user interaction and contribution as well as communication.

Thus, Web 2.x is acting as platform that embeds various forms of the services and architecture deployment.

Two-way (hype rtext based) Web, as two-way links platform. The goal of early hypertext systems and the Web 2.x environment is considering much about two-way real interactions.

$\mathbf{B} \log$, as one of the most highly touted features of the Web 2.x era, is a personal home page in diary format.

RSS. One of the things that has made a difference in ordinary Web 2.x deployment suites, is a technology called Really Simple Syndication (RSS) as an XML-based format for content distribu- 
tion. RSS is by no means a perfect format and it is the most significant advance in the fundamental architecture of the Web.

DynaWeb. Recently, there are dynamic Web sites (i.e., database-backed sites with dynamically generated content) that replaced static Web pages. What are dynamic about the live Web are not just the pages, but also the links.

Permalink, as the tool for perennially keeping Weblogs, turns Weblogs from an ease-ofpublishing phenomenon into a conversational mess of overlapping communities. The permalink was the first and most successful attempt to build bridges between Weblogs creating the space of blogosphere.

Blogosphere, as shared intellectual space inhabited by bloggers presents the community of bloggers and it is made up of blogs and bloggers. These elements that make up the blogosphere as a system of blogs are often functionally interchangeable categories.

The study of ecology that is concerned with the interrelationship of living organisms and environment is not limited to biological systems (Kurzweil, 2005), but may be found in mechanical, chemical, phys ical, physiological, social, political and economic systems (Bates, 2001). Indeed, the blogosphere may be thought of as a whole ecology even as it is a biome within the broader ecology of the Internet. Parallel to the definition of the Earth Biomes that are defined as the world's major communities, classified according to the predominant vegetation and characterized by adaptations of organisms to that particular environment (Campbell, 1996) blogosphere took the form of biomes. The blogosphere is also a social phenomenon. If an essential part of Web 2.x is harnessing collective intelligence, turning the Web into a kind of global bra in, the blogosphere is the equivalent of constant mental chatter in the forebrain. It may not reflect the deep structure of the brain, but is instead the equivalent of conscious thought. In addition, as a reflection of conscious thought and attention, the blogosphere has begun to have a powerful effect as social component. There have been many attempts to design social software in order to help Internet to survive but without any formal success. The blogosphere (which was not designed by anyone but is an emergent phenomenon) helps Internet to survive in some way.

\section{Social Networks 2.x as the Internet Portfolio}

In the process of the Web maturity, the Web experience has changed from a human interacting with a browser, to the sum of social media experiences. One consequence is that moving beyond the current notion of Web 2.x demands research advances that straddle the boundaries between computational and social sciences, the latter including microeconomics, cognitive psychology and sociology. The central principle behind the success of the Web 1.x era is based on accepting the power of the Web to harness collective intelligence with the mass of hyperlinked information. The infrastructure of the Web today much more relies on the peer-production methods through instance of collective, net-enabled intelligence. There are a lot of open projects and anyone can add a project, download and use the code of published projects, thus making new collective idea.

As technology advances, it is capable of taking on more and more advanced tasks - first simple labor and now the processing of information. As these capabilities become easier and well within everyone's reach, their value declines (Levy, 1997). However, the one thing that is beyond the reach of pure technology is the construction and maintenance of social interactions.

With new information technologies (especially the Internet) it is now possible to tie together the intelligence of huge numbers of people, connected in very different ways and on a much larger scale than has ever been possible before. People and computers could be connected so that collectively they act more intelligently than any individual, group, or computer has ever done before. Using the Web, people are finding information (Adamic \& Adar, 2005), and they are exchanging 
ideas, opinions, stories, and personal content. It is strongly connected with the mobile networks producing the new culture, demand and users' involvement in the process of architecture design Rheingold (2003) called them smart mobs with the notion on the need for internetworking standardization development.

The desire to network is as old as humanity and online social networking sites appear to solve a need that is different from simply using email, chat and blogging tools separately. Hence, the idea that there will be one big social network platform is coming on the scene. Mass communication across numerous devices and channels draws the furthest-flung reaches of our world together. People from all walks of life can now peer into the lives of others almost anywhere else in the world. Social Networks as the center must migrate towards an open, decentralized, free framework for user-centric digital identity. Since it is communication platform where many individuals are going to be presented in the some way, open, universal and secure personal identification is needed, too.

Open, universal identification platform starts with the concept that anyone can identify himself or herself on the Internet in the same way Web sites do with a Universal Resource Identifier (URI). Since URIs are at the very core of Web architecture, they provide a solid foundation for usercentric identity. Users want open standards, unified access to a combination of systems and platforms - access to everyone at any time. So, how will social networks develop in the long-term to provide access to information? The networks will become vertical in some way, appealing to specific interests or demographics - one for friends, one for family, and one for professional contacts. Such vertical networks interaction asks for open standards supporting a truly open social Web. In this context, an important aspect of the open social graph is ability to declare the different Web sites an individual use and tie them together. From the social perspective of human interactions, the natural way for people to interact within new information technologies is online communication. This notion led to the conclusion that an online network can be social and it can serve a specific purpose of a group of individuals who have something in common. Hence, social networks are taking over the Web and changing how we interact with other people and business. This social phenomenon offers creation and distribution of Web content characterized by open communication, decentralization and sharing.

The study of large-scale networks has emerged over the past several years as a theme that spans many disciplines, ranging from computing and information science to the social and biological sciences (Kleinberg, 2006). Indeed, a shared interest in network structure is arguably one of the forces that are helping draw many of these disciplines closer together. As one aspect of this broader theme, we consider a convergence of ideas taking place at the boundary between distributed computer networks and human social networks. Distributed computer networks are consisting of computing devices linked by an underlying communication medium. Human social networks are consisting of people and organizations in society connected by ties that represent friendship, interaction, and influence. Distributed computing systems have long been intertwined with the social networks that link the ir user populations. However, recent developments have added further dimensions to this relationship: the growth of blogging, social networking services, and other forms of social media on the Internet have made large-scale social networks more transparent to the general public than ever before. They have also raised new research challenges at the interface of computer science and the social sciences - challenges in which the study of distributed computing has the potential to provide considerable insight.

Guilder (2002) cited Metcalfe thought that Ethernet works in practice but not in theory. The same could be said for all the devices of the microcosm and telecosm. Both of the supreme sciences that sustain computer and communications technology - quantum theory and information theory are based on probabilistic rather than deterministic models. They offer the underpinnings for an age of individual freedom and entrepreneurial creativity (Guilder, 2002). Humankind's constant 
search for deterministic assurance defies the ascendant science of the era, which finds nature itself as probabilistic.

This scenario is seen with social networks that are taking over the Web and changing how we interact with other people and businesses. With over 1 billion people around the world now online, the future of marketing and technology will depend on the social graph that maps our connections to each other, the patterns of interaction with bus inesses and services we use, and the distribution and visibility of our shared social activities. Social Networks 1.x were built during the late 1990s to enable the initial set of consumer services that created such excitement about the promise of the early Web. Social Networks 2.x began in the early 2000s when entrepreneurs got to think about the nature of their online networks and the power that could come of making those networks explicit. New services emerged to allow users to organize their recreational and business networks. The focus of those services as they were first built was to enable the creation, growth and management of an explic it social network. In other words, the consumer experience of Social Networks 2.x was around the creation and discovery of the network itself, rather than a particular use of that network. Thus, social network becomes an important ingredient of all sorts of consumer experiences.

\section{The Future of the Internet under the Microscope}

Internet was seen in last decades through the "network effects" syndrome. It was connected with Metcalfe's Law and the presumption that new technologies are valuable only if many people use them. Specifically, Metcalfe's Law is expressed in two general ways:

1. The number of possible cross-connections in a network grows as the square of the number of computers in the network increases.

2. The community value of a network grows as the square of the number of its users increase.

Metcalfe's Law is often cited as an explanation for the rapid growth of the Internet (especially the World Wide Web). Together, with Moore's Law about the rate at which computer power is accelerating, Metcalfe's Law can be used to explain the rising wave of information technology. The most dramatic demonstration of Metcalfe's Law during the beginning of the digital age has been the explosion of the Internet in the early 1990s. The Internet had existed in various forms for many years, but reached critical mass in mid 1990s. From there, the Internet became the core information technology, attracting not only users but also business, administration, courts etc.

The more people who use software, network, standard, game, book or any other content, the more valuable it becomes, and the more new users it will attract, increasing both its utility and the speed of its adoption by still more users. These notions are strongly connected to the success of the previous Web development. The stunning success of Internet has changed the way we work, play, and learn. The Internet architecture, developed over 30 years ago, has proven its worth by the vast array of applications, and the wide variety of network technologies over which it currently runs. However, the Internet's increasing ubiquity and centrality has brought a number of challenges, for which the current architecture is ill suited.

\section{Web as Platform}

The Web computing platform encompasses today a wide range of functions, a lot of which are accessible via Application Programming Interface (APIs) - from the relatively simple bookmarking service to more complex functionality. The new Web platform is changing the game. The power of the building blocks can be leveraged to roll out complex, intelligent applications in a very compressed time frame. It is now possible to build smart browser-based and desktop appli- 
cations that utilize the power of Web Services. Because these applications do not necessarily need to worry about the backbone, they can focus more on usability, ease of use, context and semantics.

The Web platform is coping with several infrastructure building blocks based an API, which means it can be integrated as a part of other services.

\section{- Storage Services}

The storage services focus on abstracting and virtualizing memory. They allow users easily store and retrieve information.

- Messaging services

They are more complex from both a technical and business perspective. This service abstracts and facilitates secure and scalable queue-based communication between arbitrary applications.

\section{- Compute Services}

They are black-box computing activities based on grid computing technologies.

\section{- The Information Services}

These services serve huge volumes of specialized information. These include geo data, product information, etc. What is common between all these services is that they provide a simple API to a wealth of data, enabling unprecedented communication between previous ly soloed information.

\section{- Search Services}

The Search Services form a key building block of the new Web infrastructure, since search is a basic and ubiquitous component of the Web. What is particularly interesting about Web searching as Platform from a technical point of view is that it is essentially a black-box Compute Service, but specif ic to the search domain. It hints at other similar services, which offer black-box solutions to sorting or data transformation problems, for example.

\section{- Web services}

These specialized services are customers of the other building blocks. They offer clear, specific and simple APIs to lookup and change the information that each owns. They are organic in nature that it makes sense to group them together as building blocks.

One other point to consider with all of this is the significant changes ahead in the enterprise. With the proliferation of broadband and the thought that the consumer is also an enterprise user, we must be cognizant of the many opportunities for Web-based services to be brought into the corporation from the ground-up. Everything we did in various desktop office and other enterprise applications several years ago can be done through a Web-based office, email, e-dating, and calendaring. The advent of the Web opened up vast banks of information to anyone with an Internet connection. Instead of treating the Web just as a handy way to publish information, businesses need to start acting like software companies and encourage programmers to build services on top of their platforms.

\section{Cloud Computing}

We are witnessing a shift in information technology that affects not only business models, but also the underlying architecture of how we develop, deploy, run and deliver applications. This shift has given a new relevance to ideas such as cloud computing and utility computing. While utility computing often requires a cloud-like infrastructure, its focus is on the business model on which providing the computing services are based. 
Utility computing relates to the business model in which application infrastructure is delivered. While cloud computing relates to the way we design, build, deploy and run applications that operate in a virtualized environment, sharing resources and boasting the ability to dynamically grow, shrink and self-heal. Cloud computing is a broader concept than utility computing and relates to the underlying architecture in which the services are designed. It may be applied equally to utility services and internal corporate data centers. Although it is difficult to come up with a precise and comprehensive definition of cloud computing, at the heart of it is the idea that applications run somewhere on the "cloud" over the network.

Cloud computing resides between Web 2.x and Web 3.x platforms as the development stage of the Web mashups. Cloud computing is a computing paradigm in which tasks are assigned to a combination of connections, software and services accessed over a network. This network of servers and connections is enabling user with on-demand computing. This vast processing power is made possible though distributed, large-scale cluster computing, often in concert with server virtualization software and parallel processing. Thus, in cloud computing the network becomes the supercomputer. Cloud computing is generally correlated to grid computing and utility computing, both of which have existed in Web 2.x era. Like grid computing, cloud computing requires the use of software that can divide and distribute components of a program to thousands of computers. New advances in processors, virtualization technology, disk storage, broadband Internet access and fast, inexpensive servers have all combined to make cloud computing a compelling paradigm. Cloud computing allows users and companies to pay for and use the services and storage that they need, when they need them and where they need them. This shift will put data centers and their administrators at the center of the distributed network, as processing power, electric ity, bandwidth and storage are all managed remotely.

\section{Web 3.x: Ongoing Evolution of the Web}

Some Web 2.x services evolve into Web 3.x services with an additional layer of individual excellence and focus. Web 3.x is a return to what was great about media and technology before Web 2.x: recognizing talent and expertise, the ownership of ones words, and fairness. Therefore, bringing together Open APIs and scraping/mashup technologies gives us a way to treat any Web site as a Web service that exposes its information - the information becomes open. In turn, this enables software to take advantage of this information collectively, and the Web truly becomes a database that can be queried and remixed seamlessly.

A prime scope of a Web 3.x technology is open concept and open platforms. The idea is that a Web site or device, like a mobile phone, should be able to accommodate whatever features or applications its user wants - the Web will become a place where much more mixing and matching of different services will be permitted. It is presented in recent days with the Android platform that was built from the ground-up to enable developers to create compelling mobile applications that take full advantage of all a handset has to offer, and it is built to be truly open. Android breaks down the barriers to building new and innovative applications. A developer can combine information from the Web with data on an individual's mobile phone to provide a more relevant user experience. Applications based on Android platform could enable users to view the location of their friends and to be alerted when they are in the vic inity giving them a chance to connect. In that way, mobile phones become the nodes within social networks. As many Web 3.x software complexity and cost is increasing, it is happening also with the mobile software. However, mobile users want the same applications as they have on the Internet, and Android will be able to deliver it. With many innovations in positive technologies, the Web is going to be much more immersive and a much more multi-dimensional environment. 
Web 3.x as a term, which definition is not unified, includes transformation of the Web into a database, a move towards making content accessible by multiple non-browser applications, the leveraging of artificial intelligence technologies, the Semantic Web, or the Geospatial Web. In the context of Semantic Web, Web 3.x is an evolving extension of the World Wide Web. In Web 3.x space, Web content can be expressed not only in natural language, but also in a form that can be understood, interpreted and used by software agents, thus permitting them to find, share and integrate information more easily. Some of the original motivations for the Semantic Web came from the failures in early Web applications that cause the problems for search and browsing in Web 2.x applications. Latent Semantics, the attempt to "mine" meaning from the words in Web content, is always problematic due to ambiguity and polysemy. For applications that wanted to share information that was not yet in textual form, or was in a form where the textual information was hard to extract, it was clear that some form of knowledge representation was needed. This was not a new observation; it had been realized in fields like Natural Language Processing and machine translation years earlier. What was new in the Semantic Web technologies was an attempt to do know ledge representation in a form that was Web embedded. The key was to create another web graph, this time a graph between semantic terms and between these terms and what they described.

A recent development of Semantic Web technologies has been occurring in the Web 3.0 technologies. In these systems, an attempt is being made to exploit more of the link spaces coupled with capturing some of the social dynamics of Web 2.0 applications (Hendler \& Golbeck, 2008). One difference between these and earlier AI systems is the attempt to figure out how to exploit the increased value of the network effect that can come from using Semantic Web technologies to provide links between diverse sets of content or users. The Web is an interesting place for browsing, but its real power derives from people finding what they need. Similarly, using Semantic Web technologies, social networks, and terminology to label and link content will be powerful only when it enables people to do powerful things. Creating these links is a first necessary step, and the research challenges lie in understanding how to use them.

Location and time extend content relevance in Web 3.x. Intimate connections are made between the real world and the Web. Combined with the social Web 2.x services, Web 3.x provides the Internet user with the opportunity to engage with people all over the world on a variety of devices (information appliances). The richness of these communications and the relevance of locally available information are what add depth to Web 2.x services, creating a Web 3.x environment. This new environment needs more flexible, more mobile, secure and ubiquitous network infrastructure opening the space for Internet 3.x development.

Web 3.x becomes highly specialized information store, moderated by a cult of personality, validated by the community, and put into context with the inclusion of meta-data through widgets based on microformats. The first step towards a Web 3.x is the emergence of the Data Web as structured data records that are published to the Web in reusable and remotely indexable and queryable formats. It is the realization and extension of the Semantic Web concept. Web 3.x extends a convergence of Service-Oriented Architecture and the Semantic Web, and it opens the new form of society through the social networks.

\section{Social Networks 3.x: Communications Impact}

Social networking becomes content-centric evolving from social networks 1.x based on the initial set of consumer services to the social networks 2.x based on creation and management of an explic it social network, and finally to social networks 3.x that are based on pure communication. Social networks 3.x embed the communications layer opening the age of ubiquitous communication. 
Social networking will be a crucial element of virtually all online consumer experiences going forward. These issues open the social Networks 3.x era - the era of social networks that include ubiquitous integrating profiles and relationships on open platform. It would be aimed toward universal identity ensuring individual to be mobile and independent as much as possible. Thus, Social Networks 3.x platforms incorporate strong decentralization with full mobility in communication activities independent of platform, standard and architecture used for those activities. The vital component of any social network success is the communications layer embedded within social network - as in early days of Internet when communications was in front of information. It is something like Retro Internet introducing some new services that are just replica of old, well known services. It should be noticed that social networks are just one layer of functionality for next-generation enterprise portals. They are implemented in the context of other knowledge discovery and management functions (Raghavan, 2002). Some researchers stated findings that computer-mediated communications can break down hierarchical and departmental barriers, standard operating procedures, and organizational norms. Some observations (Galagher, Sproull, \& Kiesler, 1998) supported the theory long popular among online enthusiasts that people who often dominate conversations face-to-face, because of rank or aggressive demeanor, are no more visible than those who would remain silent or say little in a face-to-face meeting but say a lot via computer-mediated communication. Businesses are the next organizations to be subjected to the same new kinds of social forces that were experienced by the research and academic communities when they went online. Kiesler (Sproull \& Kiesler, 1992) offered evidence that people communicate across and around traditional hierarchical organizational boundaries if their mutual interest in a particular subject matter is strong enough. They approved that groups make more daring decisions via computer-mediated communication (CMC) than they do face-to-face, and work that later turns out to be important is sometimes accomplished in informal conversations as well as in structured online meetings.

\section{New forms of Internet Survival Kit}

\section{Web 2.x as Placebo}

Web 2.x technologies are presenting to most of business users as real Internet survival solution that could act as placebo. In fact, placebos originated as sugar pills given to patients who might or might not get better simply by believing the pills would work. Today, a placebo is (generally) a pill that looks just like a real medication but has no active ingredients. It is used in clinical trials to test the effectiveness of the medication. If the medicine is in liquid, powder or capsule form, the placebo takes the same form. In the same way, Web 2.x technologies took the form of the current Internet illness thus making an impression of Internet illness recovery making it useful for modern business services and applications. Most of current and future services and informationcommunications systems applications need healthy communications environment with stabile (robust) addressing scheme, mobility and ubiquity. Not all of these needs are satisfied with the Web 2.x technologies.

\section{Placebo Effect of Web 2.x Technologies}

The placebo effect of Web 2.x technologies is the measurable, observable, or felt improvement in information-communications systems health not attributable to treatment. Many people believed that this effect exists due to the placebo itself in some mysterious way. Some believe the placebo effect is psychological, due to a belief in the treatment or to a subjective feeling of improvement. However, it may be that much of the placebo effect is not a matter of mind over molecules, but of mind over behavior. There is a certain amount of role-playing by ill or hurt people, which use the 
Web 2.x technologies as the vademecum for doing business and services. The behavior of such injured persons is driven mostly by social and cultural factors. However, the informationcommunications systems based on recent Web technologies have no real potential to rescue Internet from illness caused by new demand and business requirement. Pervasive and ubiquitous computing environment asks for real survival instrument that will make Internet as universal tool capable for interconnecting a huge number of information appliances and femtocells units across the globe. Besides many attempts to prolong reconstruction of Internet architecture, Web 2.x technologies take the form of acceptable solutions to supply new services in the communities where business and ordinary users are not so familiar with the potential of information technology and information-communications systems utilization.

\section{Internet Survival Kit}

We often think of the Internet as a platform for independent global communication, where information flows freely, innovators can launch new applications at will, and everyone can have a voice. However, it is likely that next generations will see Internet as different medium. Furthermore, there will be poss ibility for scenarios in which Internet, as we know today will disappear. One scenario for Internet death could be the end of net neutrality where no one would be able to launch the new services without the tacit approval of carriers. Many countries already restrict how the Internet is used. However, the Internet is a tool for social change and revolution that could threaten any government. Since these actions are against users' premises, the Internet could die soon. This notion is in the context of the Internet as the medium and the fact that there is no the Internet without users. The Internet has already morphed from its initial aspirations of open academia to a commercial platform controlled by corporations and carriers.

New way to keep Internet live is with the news and media diversification across the net. The publisher can provide related clips that would be of interest of the viewer, thus engagement with online video news is deeper and longer. This offers a bit of confirmation to a theory that the Internet will reverse dumbing down news that was caused by television. One reason that television reduces most news segments to one minute is that everyone watching a newscast has to watch all of them, even the topics that bore them. Video on the Internet is more like reading a print publication. The users decide how much they want to watch and when to switch to the next topic. Furthermore, now anyone can publish his or her writing and upload video to the Internet. It used to be if you wanted to become a radio media, you had to convince a radio station to give you airtime. Now you can record your own radio podcast. This same effect is why some Web optimists see the opportunities brought by the social, participatory Web as mainly a good thing.

Carr identifies big switch to a global Web computer (Carr, 2008) - but Internet of today should be aimed toward big digital personal video recorder platform. While it may seem that we are in the middle of an unprecedented technological transition, Carr assumes that the direction of the digital revolution has a strong historical corollary: electrif ication. Carr argues that computing, no longer personal, is going the way of a power utility. We are in the midst of a similar transition in computing, moving from private local hard drives to the computer as access portal. Soon all companies and individuals will outsource their computing systems, from programming to data storage, to companies with big hard drives. The social and economic consequence of this transition into the utility age is almost near. Wealth will be further consolidated into the hands of a few, and specific industries, media/publishing in particular. This scenario leads us to the Internet as global personal video recorder, the new global utility. It opens the horizon for home systems maturation and the additional way to stop Internet dying.

The Internet plays the potential impact on computer, network, and communications systems, and the effects of these changes on the systems that rely on the Internet. Internet to survive must be 
extremely penetrative. It is a story with a long history and many parts that move in fits and starts, weaving cycles with beginnings and endings. Internet 1.x was about the network. Internet 2.x has been about devices using that network. Internet 3.x will be about the software weaving together the devices living on top of the network. It also will be about how users with these devices will be able to communicate seamlessly with each other and use systems resources more effectively across geographies and operating platforms (Kumar et. al, 2005). Internet 3.x features many of the same participants as Internet 2.x; it also highlights several emerging innovations and technologies. Intelligence of the network will continue to migrate to edge devices, (i.e., devices as close as possible to an end-user rather than a central resource). Networks will continue to expand and much of their functionality will be distributed and decentralized. While the architecture of the current Internet and Web will continue to be relevant, Internet 3.x will involve significant architectural changes.

The survival cycle of the Internet 3.x begins with the deployment of distributed and decentralized architectures. Already, the laws of network dynamics indicate that the client devices will be thicker in the future because the cost of decentralizing (i.e., localizing) content, processing, and data on the client device will be cheaper than centralizing assets and resources. Client devices will be able to do more consequently. The key (core) players in creating Internet survival kit are distributing and decentralizing (DI-DE) actors.

\section{DI actors}

Distributed processing - is defined as the technique by which large processing tasks are segmented into component blocks, de livered to a number of devices where these blocks are processed, and reassembled by a central device. The fundamental idea behind distributed processing is that more devices can process more data faster. Distributed processing technologies allow several processing devices (such as servers or personal computers) to be linked together to share processing resources. The current information technology development makes the shift to a new software model based on heterogeneous collections of cores optimized for various tasks. Such multicorebased computers should be more like cellphones, using a variety of specialty cores to run modular software scheduled by a high-level applications programming interface. Multicore design will deliver a server farm on a chip, shrinking the size of data centers. It will reduce the need for complex distributed data processing architecture over Internet zones. Distributed processing is also mostly connected with the term of parallel processing that for the Internet survival kit constitutes a key factor.

Distributed Storage Services - as a network topology in which storage assets are reshaped into components and distributed across geographies in order to create a clustered, wide-area storage network. Freeing data from geographic isolation and expensive equipment and maintenance costs is a significant value proposition. While storage equipment costs are going down, the most significant portion of a storage infrastructure's costs reside in software and maintenance/services.

Distributed Network Se rvices - are defined as intelligent network services that accelerate the delivery of data, propagate data more efficiently, or minimize the cost of operating existing network services using distributed resources across geographies. They are mostly based on decentralized network concept.

\section{DE actors}

Decentralized network - Centralized client-server is by definition directional and hierarchical. Directionally, a client device pulls data from a server and generally stores data onto servers. Hierarchically, the client connects to a server and is subject to the control and administration of server resources. The Centralized client-server architecture is ideal for many applications and tasks cop- 
ing with the information technology in the past and current days. For example, central management of resources has been a hallmark of secure networks. Network file management and collaborative applications have benefited tremendously from stable and secure central management. However, the topology of the Centralized client-server network inevitably yields ineffic iencies, bottlenecks, and wasted resources. Most enterprise applications and hosted services on the Internet require high capital expenditures and maintenance costs. Decentralized systems are about making the router the central point of the network, and forcing processing, storage, and intelligence to edge devices. If the server is squeezed out of the route, then processing, storage, and intelligence must reside on client devices. In a Decentralized client-server model, since the server is removed from the client-server-client transaction scheme, client devices can communicate directly with other client devices.

Unified Communications - There is a huge burden on communication because so many people conduct day-to-day activities from almost anywhere. People already use a variety of devices to communicate, including wire less phones, personal digital assistants, laptops and smart phones with messaging, instant messaging and text messaging capabilities. In addition, Internet Protocol based (IP) phones and the Session Initiation Protocol (SIP) based multimedia tools pull together faces, voices, documents, and presentations into a single virtual, collaborative space. It is about the unified communications concept that means breaking down barriers so that people using different modes of communication, different media, and different devices can still communicate with anyone, anywhere, anytime. For example, with its SIP-based architecture, a unified communication platform removes dependence on location. Unified communications technology provides the power to reach people almost anywhere, at any time, and provides the flexibility to allow people to control when they can be reached. Unified communications encompasses several communication systems or models including unif ied messaging, collaboration, and interaction systems; real-time and near real-time communications; and transactional applications. Unified messaging allows users to access voice, e-mail, fax and other mixed media from a single mailbox independent of the access device. Multimedia services include messages of mixed media types such as video, sound clips, and pictures, and include communication via short message services. Collaboration and interaction systems focus on applications such as calendaring, scheduling, workflow, integrated voice response, and other enterprise applications that help individuals and workgroups communicate efficiently. Real-time and near real-time communications systems focus on fundamental communication between individuals using applications or systems such as conferencing, instant messaging, traditional and next-generation private branch exchanges, and paging. Transactional and informational systems focus on providing access to m-commerce, e-commerce, voice Web-browsing, weather, stock-information, and other enterprise applications. In these communications process, the presence catches the social networks' streams. Whereas unified communications is about blending different communications media, presence is the engine on which unified communications runs.

Decentralized Collaboration - is defined as a peer-to-peer system for real-time communications that facilitates the creation of workgroups in which the user's device and its assets become central. In this context, collaboration means a decentralized cooperation across thousands or even millions of Internet users - as opposed to the traditional individuals working together that we usually think of when we talk about collaboration. Decentralized collaboration generally includes such features as instant messaging, file sharing/transfer, co-browsing, and personal information sharing. Friedman stated (Friedman, 2007) that cheap, ubiquitous (and decentralized) telecommunications have finally obliterated all impediments to international competition creating 'flat world' - advances in technology and communications put people all over the globe in touch as never before. 
One of the more powerful ideas of decentralization in Internet 3.x is the emphasis on the primacy of users' ownership and control of data and resources. The only two assets that matter in a system are the data and the system resources associated with the user. Decentralization liberates the user from the grip of central management, especially when it is advantageous to both central management and the user. Decentralization frees data to move, as users deem most appropriate, and emancipates system resources for maximum utilization. Here is the place for microformats that offer machine-usable data while at the same time providing usable and presentable content. They are also capable of adapting to current behaviors and usage patterns giving to the network more dynamics. The users are in possibility to share new experience with microformats that solve the specific content presentation problem.

Microformats as a set of simple, open data formats built upon existing and widely adopted standards (Khare, 2006) are based on the concept that underlines the user-centric aspect rather than making information interpretable by machines. It presents the step further on the road to the full and usable Semantic Web.

As bandwidth capacity continues to grow, the compute-to-data ratio will continue to grow. That is, when bandwidth becomes so abundant that the cost of transmitting large blocks of data is marginal, processing will be the more valuable resource, and processing resources will demand an even greater premium. In such an environment, it makes even more sense for a system to take advantage of installed processing resources distributed across networks.

The Internet's developing third generation appears poised to exploit technological innovations, expanding broadband access, wireless and converging markets with even greater service diversity and market segmentation. This is the place where the Web 3.x acts as a survival method because computing of any kind starts with infrastructure. Web 3.x involving the decentralization of digital data, software and infrastructure into modular components provides the opportunity for reintegration of existing applications into new applications or functions on the fly by either machines or people.

This notion takes into account the affect that utility computing will have on the rest of Web 3.x. The process of decentralization and disintegration of the infrastructure into modular components entails defining the specific Web 3.x platform. Thus, modularity and composability are side effects of platform standardization that is crucial for commodity components (for Web modules that can be combined freely). Composability, as a system design principle that deals with the interrelationships of components, presents another tool in Internet survival kit.

In the process of Internet development, we must admit that a critical part of our ability to see and perceive is not logical, but emotional. Therefore, for computers to have some of the advanced abilities we desire, it may be necessary that they comprehend and, in some cases, feel emotions. Emotion is fundamental to human experience, influencing cognition, perception, and everyday tasks such as learning, communication, and even rational decision-making. However, technologists have largely ignored emotion and created an often-frustrating experience for people, in part because affect has been misunderstood and hard to measure. The aim should be to restore a proper balance between emotion and cognition in the design of information technologies for addressing human needs. Thus, there is postulation (Picard, 1997) that new systems have a strong demand on designing new ways for people to communicate affective-cognitive states to machines. It means creating new techniques to assess frustration, stress, and mood indirectly, through natural interaction and conversation, and making information appliances within ubiquitous computing environment more emotionally intelligent. 


\section{Concluding Remarks}

The Internet 3.x encompasses seamless mobility enabling the user to be able to access various media, data, and information anywhere, anytime. As the user move from one network to another, the communication and transmission are not interrupted. It is switched to the appropriate network, and at that time, the user does not notice the change. Thus, Internet 3.x as the survival kit of information-communications systems must incorporate full convergence that takes voice and all the multimedia services together and putting them into different networks and devices. In that process, the wireless is the one technology that does challenge the original Internet architecture - mobility itself is the key requirement for future information-communications systems architecture.

Advances in wireless mobile network technologies and mobile information appliances facilitate infrastructure that can support a range of mobile services and applications in addition to conventional Internet access. These network systems provide users with cost-effective services that have the potential to run anywhere, anytime, and on any device without (or with little) user attention. Driven by the equally rapid advance of mobile terminals, end-user demand for mobile information appliances is increasing. The one of possible steps toward the design of informationcommunications systems survival kit is considering high availability applications and network services. High availability applications and network services that usually run on computers that are more powerful allow users to enjoy the freedom of mobility, network neutrality and personal media production.

\section{References}

Adamic, L., \& Adar, E. (2005). How to search a social network. Social Networks, 27(3), 187-203.

Bates, D. G. (2001). Human adaptive strategies: Ecology, culture and politics. Needham Heights, MA: Allyn and Bacon.

Campbell, N. A. (1996). Biology. Menlo Park, CA: Benjamin/Cummings Publishing.

Carr, N. (2008). The big switch: Rewiring the world, from Edison to Google. New York: W. W. Norton.

Fried man, T. L. (2007). The world is flat. New York: Farrar, Straus and Girou x.

Galagher, J., Sproull, L., \& Kiesler, S. (1998). Leg itimacy, authority, and community in electronic support groups. Written Communication, 15, 493-530.

Gu ilder, G. (2002). Telecosm: The world after bandwidth abundance. New York: Simon \& Schuster.

Hendler, J., \& Golbeck, J. (2008). Metcalfe's law, Web 2.0, and the Semantic Web. Web Semantics, 6(1), 14-20.

Khare, R. (2006). Microformats: The next (s mall) thing on the Semantic Web? IEEE Internet Computing, $10(1), 68-75$.

Kle inberg, J. (2006). Distributed social systems. In J. Aspnes (Ed.), Proceedings of the 25th ACM Symposium on Principles of Distributed Computing, pp. 5-6. New York: ACM Press.

Ku mar, R., Liben-Nowell, D., Novak, J., Raghavan, P., \& To mkins, A. (2005). Theoretical analysis of geographic routing in social networks. Technical Report MIT-LCS-TR-990. Cambridge, MA: MIT Pres.

Kurzweil, R. (2005). The singularity is near: When humans transcend biology. New York: Viking Penguin.

Levy, P. (1997). Collective intelligence: Mankind's emerging world in cyberspace. New York: Plenum Publishing.

O'Reilly, T. (2005). What Is Web 2.0? Design patterns and business models for the next generation of software. Retrieved Septe mber 30, 2005 from http://www.oreillynet.com/pub/a/oreilly/tim/news/2005/09/30/what-is-web-20.ht ml 
Will Information-Communications Systems Survive?

Picard, R. W. (1997). Affective computing. Ca mbridge, MA: MIT Press.

Raghavan, P. (2002). Social networks: from the Web to the enterprise. Internet Computing, 6(1), 91 - 94.

Rheingold, H. (2003). Smart mobs: The next social revolution. New York: Basic Books.

Sproull, L., \& Kiesler, S. (1992). Connections: New ways of working in the networked organization. Cambridge, MA: MIT Press.

\section{Biography}

Fjodor Ruzic is doctor of information sciences at University of Zagreb where he is lecturer in

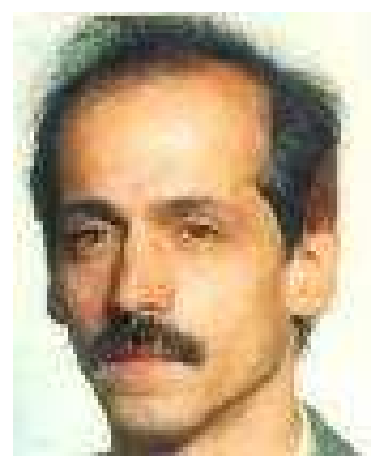
new media, and interactive multimedia systems. His recent research activities are covering the integration of information content, infrastructure and information appliances. He is in information sciences from 1975, and he is member of many national and international bodies relating to telecommunications systems integrity, information resources management and multimedia system environment. He was working on research and implementation of networked databases, educational material, and digital media. He published over 125 scientific and research papers in various international journals and he is author of several books dealing with graphical user interfaces, multimedia and Internet. 